\title{
A Novel Human Computer Interaction Paradigm for Volume Visualization in Projection-Based Virtual Environments
}

\author{
Changming He, Andrew Lewis, and Jun Jo \\ Griffith University, School of Information and Communication Technology, Australia \\ \{c.he, a.lewis, j.jo\}@griffith.edu.au
}

\begin{abstract}
We propose a novel Human Computer Interaction (HCI) paradigm for volume visualization in projection-based immersive virtual environments (VEs). This paradigm is intuitive, highly efficient and allows accurate control over the virtual objects. A fine control mode for direct manipulation is proposed to address the low accuracy problem of virtual object manipulation in VEs. An agent object interaction method is proposed to provide more flexibility in manipulating the volume objects. A two-handed scaling method is proposed to conveniently scale the volume object along one, two, or three axes. Finally, a ghost object paradigm is proposed to address the motion constraint problem for virtual objects. An implementation using a 3-state tracked glove setup as the input interface is discussed. How basic functionality for volume visualization can be transferred from the 2D WIMP (Window, Icon, Menu, and Pointer) interface to a 3D VR interface is also systematically discussed.
\end{abstract}

Keywords: human computer interaction, virtual reality, volume visualization

\section{Introduction}

Volume visualization, which allows users to see and explore the internal structure of 3D data sets, is one of the most important forms of scientific visualization. However, the nature of volumetric data makes it a great challenge to display all hidden 3D patterns within the data. Researchers have proposed different volume rendering algorithms, such as iso-surface extraction [1] and direct volume rendering [2], to display these patterns. Various types of transfer function specification methods [3] have been proposed to highlight regions of interest and filter out extraneous details. Virtual Reality (VR) technology has been used to enhance the depth perception of the volume data $[4,5]$.

Compared with conventional 2D computer displays, virtual environments (VEs) provide two additional depth cues: stereoscopic vision and motion parallax. VEs have been proven to be better media to convey 3D structure to users [6]. VEs provide users with environments that are much closer to the real world, so the interaction paradigms for VEs are fundamentally different from those designed for $2 \mathrm{D}$ environments. Interaction in 3D virtual environments requires control over 6 degrees-of-freedom (DoF), in contrast with 2 DoF for 2D environments. More degrees of freedom provide 
many more possibilities for advanced interaction methods that can improve communication between human and computer, but simultaneously require much more effort in designing the 3D interface.

Researchers have proposed various HCI paradigms [7-11] for general virtual object interaction. Some other researchers proposed different volume visualization systems in VEs (see, for example, $[5,12,13]$ ). However, few have systematically addressed how to transfer all the basic functionality for volume visualization from $2 \mathrm{D}$ interfaces to VR interfaces. This paper addresses this problem by proposing a HCI paradigm for volume visualization in projection-based VR.

\section{Interaction Design}

The proposed HCI paradigm was implemented in the Complex Active Visualization Laboratory in Griffith University. A $5 \times 2$ metre polarizing screen and 4 rear projectors equipped with polarizing filters enable the stereoscopic capability. A Polhemus FASTRACK system is used to facilitate the 6 DoF motion tracking requirements for VEs. The design of the HCI paradigm is part of the Virtual Reality Volume Visualization (VRVolVis) project, which is attempting to develop an intuitive volume visualization VE with several, novel, volume data exploration tools.

Projection-based VEs have a greater Field of View (FOV) and Field of Regard (FOR) than desktop-based VEs and can provide users with life-size perception of the virtual objects. Compared with Head Mounted Display-based (HMD-based) VEs, projection-based VEs have lower end-to-end delay requirements of the systems, and generate less VR sickness. For these reasons we believe that projection-based VEs are well suited for frequent use for volume visualization purposes. It should be noted, however, that though the proposed HCI paradigm is for projection-based VEs, it can be adapted easily to HMD-based VEs.

\subsection{The Basic Interaction Tasks for Volume Visualization}

After analyzing a number of visualization systems, such as Volview [14] and Simian [15], four types of basic interaction tasks needed for volume visualization were identified. These are:

- volume object transformation,

- volume exploration,

- transfer function specification, and

- system control.

Volume object transformation includes translation, rotation and scaling of the volume data. Volume exploration is the task of using virtual tools such as a clipping plane to study the internal structure of the volume data. These two types of interaction tasks involve 6 DoF control over the virtual objects, which is naturally provided by immersive VEs. VEs potentially provide a much better interface for these tasks.

Many different methods for transfer function specification have been proposed [3]; all of them use 2D WIMP interfaces. Trial-and-error is one of the most popular methods, and is used by commercial software such as Volview[14]. Volview provides 
three separable TF settings, which map voxel values to opacity, voxel values to color, and voxel gradient magnitudes to opacity. This interaction to adjust the TF is essentially moving multiple control points on a plane. System controls for volume visualization include loading or saving a volume to the system, configuring simple states, setting simple parameter values, etc. Both TF setting and system controls have worked very well in the WIMP interface. In this work we propose a paradigm that can transfer most 2D WIMP interface functions into a 3D immersive environment.

\subsection{The input interface and its visual feedback}

Guiard proposed a framework for classification of interaction tasks [16], which is well summarized by Haan et al [17]. Various types of hand-involved interaction tasks can be grouped into three categories:

1) One-handed task (only one hand performs a task);

2) Double one-handed task (each hand performs a separate one-handed task);

3) Two-handed task (both hands cooperate to perform a single task). The category of two-handed task includes two subclasses:

a. symmetric two-handed task (both hands perform the same actions), and

b. asymmetric two-handed task (each hand performs different actions involving complex coordinate between hands). Asymmetric two-handed task is the most common form of two-handed task.

An effective interface for VEs should allow users to interact with the virtual world the way they interact with the real world. Therefore, it should support all of the interaction methods mentioned above and have the following features. Firstly, the interface should allow users to use either left or right hand to manipulate objects (support one-handed tasks). Although almost all users do have hand preference (handedness), for many simple tasks either left or right hand would be used, depending merely on which hand is closer to an object. Secondly, the interface should allow users to simultaneously manipulate two different objects (support double one-handed tasks). Finally, the interface should support two-handed tasks in which users employ two-hand interaction to achieve a goal. For some tasks, such as resizing a volume object, using two-handed interaction is much more natural than using one-handed interaction.

Several researchers have used the "pen and pad" metaphor to interact with projection-based VEs [17, 18]. A physical, transparent pad is held by the non-dominant hand to provide a frame for the interactions, and a pen-shaped stylus is held by the dominant hand to perform fine interactions. One of the advantages for this interaction paradigm is that passive, haptic feedback is provided to the users. However, this interaction paradigm has a few disadvantages:

1) The mismatch of the focal depth of the virtual and real panels will cause discomfort; and

2) The hands' activities are limited by the physical tools, so the possible interaction methods are also limited.

Instead of interacting with virtual environments via hand-held physical objects the proposed interaction paradigm projects the users' hands into the virtual world and 
allows the users to directly manipulate various virtual objects.

To manipulate small objects in real environments, the thumb and index finger are usually sufficient. Most purely-visual virtual environments are mass-less (without inertial effect) and agravic (zero-gravity), so thumb and index finger are considered sufficient for manipulating virtual objects. The input device proposed is a pair of tracked gloves. The right hand setup is shown in Fig. 1. Six DoF motion trackers are attached to the thumb and index finger to track their positions and orientation. A switch is attached to the thumb, and this is used for the "apply" status for some of the tools. This approach provides three unambiguous states for the fingers: open (fingers separated), closed (fingers just touch each other), and apply (when force is exert to close the switch). For many tools, such as the clipping widget, two states (open and closed) are sufficient, because the fingers can be closed to grab the tool, applying it to the volume, then opening the fingers releases the tool. Adding the "apply" state enlarges the vocabulary for interaction. For example, a function "cross-section snapshot" could be added: once a user presses the switch while using the clipping widget, a cross-section of the volume can be taken out for detailed study.

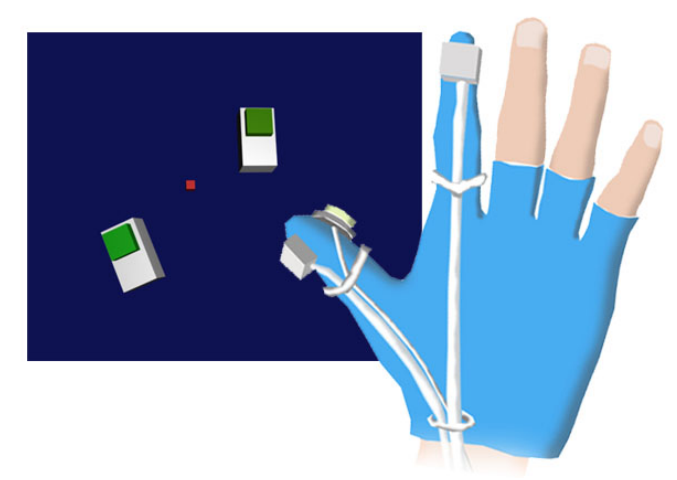

Fig. 1. The tracked glove setup and its visual feedback

When a user wears these gloves, the $6 \mathrm{DoF}$ of the thumb and index finger are continuously updated, and two virtual hands are shown in the virtual space. The user can manipulate virtual objects via these virtual hands. A simple and unambiguous representation of the virtual hands is advocated: instead of rendering the whole hand, just the thumb and index finger are drawn. There are a few reasons for this:

1) To render the whole hand, more tracking and more graphical calculation would be needed;

2) It is not necessary to show the whole hand because just thumb and index finger are used to manipulate virtual objects; and

3) Drawing only thumb and index finger can minimize problems with occlusion.

Two cuboids are used to represent each finger, as shown in Fig. 1. The larger cuboid indicates the position and orientation of the finger, and the smaller cuboid is used to indicate two things: the nail's position (the sensor position) and the finger state. Showing the nail's position further clarifies the fingers' orientation. The status of the fingers is indicated by the color of the nail box: green indicates that the fingers 
are opened; yellow indicates that the fingers are closed; and red indicates the "apply" state. A tiny cube is drawn at the midpoint between the thumb and index finger. The position of this cube is the position that the fingers will grab when the fingers are closed, so this cube can provide subtle occlusion depth cues to the users and inform them whether the grab point is within, or outside of, a virtual object.

\subsection{Basic Interaction}

Bowman [19] classified VR interaction into four categories: viewpoint control; object selection; object manipulation; and system control. Following this classification, this section discusses the basic interaction methods proposed for volume visualization in projection-based VEs.

Viewpoint Control. Researchers have proposed various techniques or metaphors for viewpoint control for VEs. For example, Ware [7] proposed the viewpoint at hand, scene at hand and flying vehicle metaphors, and Stoakley [8] proposed the WIM (worlds in miniature) interaction metaphor. Unlike some VE applications that have large virtual spatial extents (such as a virtual theme park), the extents for volume visualization applications are usually not too large, so large scale viewpoint translation is not necessary. VRVolVis generates a virtual space that extends about one meter in front of and a few meters behind the projection screen. View point control is achieved through walking and head movement, which is actually a key component of VR technologies. A user can freely change the viewpoint this way to exploit the active motion parallax depth cue, which has been proved to be an important depth cue for people to understand 3D structures [6].

Object Selection and Manipulation. Researchers have proposed various metaphors or techniques for object selection and manipulation in VEs. Mine [11] believed the direct manipulation metaphor is better than arm-extension techniques because direct manipulation allows the user to exploit proprioception in interaction. However, since direct manipulation has a reachable range limited by arm length, he proposed the scale-world-grab techniques. Some researchers believe that the scale of the virtual scene should remain stable, otherwise it might confuse the user [20]. Arm-extension techniques like the Go-Go interaction technique [9] extend the reachable range of a user. For a large-scale virtual scene, ray-casting [21] or image-plane selection techniques [10] can be used.

Since volume visualization is generally of limited range, it is believed that direct manipulation is suitable. In HMD-based VEs, where the real-world is totally blocked, the virtual hand can overlap with the real hand and thus proprioception can be exploited, but in projection-based VEs, overlapping real and virtual hands causes problems. When a virtual object is closer to the eyes than the virtual hand, the virtual object should occlude the virtual hand, but in projection-based VE the screen is actually behind the real hand, so correct occlusion effects cannot be achieved. This visual conflict between two major depth cues - occlusion and convergence (a strong depth cue when the scene is close to the user) - will destroy the perception of immersion, and produce significant discomfort. Fixed z-axis offset techniques can 
help to solve these problems by placing the virtual hands in the space, where $\mathrm{x}$ and $\mathrm{y}$ coordinates follow the real hands while the $\mathrm{z}$ coordinate has a fixed offset (from 0.5 to 1.0 metres).

To select and manipulate a virtual object, the user moves the virtual hand to the object, and then closes the thumb and index finger to select the object. Once the object is selected, the object will follow the virtual hand (which means that the relative position and orientation remain constant, as if the virtual object is firmly held by the virtual hand). When rotating a virtual object in space, normally one motion is not enough. In this case the ratchet rotation method can be used. Just like using a ratchet motion to rotate a knob around its axis in the real world, a user can open the fingers, return the hand to a start position, close the fingers and repeat the rotation and the rotation will be accumulated. To achieve a certain spatial angle for a virtual object, a few ratchet motions may be needed, but since manipulation is very similar to real world object manipulation, users need little time to learn to use this method.

Mine [11] pointed out that one of the limitations of VEs is that precise manipulation of virtual objects is difficult. There are a few reasons for this. Firstly, the tracking system is not accurate and stable enough. Most of the motion tracking systems that are based on ultrasonic, optical or electromagnetic tracking have limited precision and show appreciable jitter. Secondly, humans depend on haptic feedback and physical constraints for precise interaction in the real world. In virtual environments, usually there is no physical constraint to guide the interaction.

To address the accuracy problem for virtual object manipulation in projection-based VEs, a fine control mode is proposed (in contrast with the direct manipulation mode) to provide finer translation and rotation control over the virtual objects. When a user translates a virtual object in the direct manipulation mode, the virtual object's displacement will be equal to the virtual hand's displacement. When translating a virtual object in the fine control mode, the displacement of the object will be a fraction of the displacement of the virtual hand's. In the same manner, in the fine control mode angular displacement of the volume object will be a fraction of the angular displacement of the virtual hand's. When interacting in 3D space, unsteady hand movement is unavoidable but the fine control mode will reduce its effect and enable a more accurate object manipulation method to the users.

System Control. System control itself is not part of volume visualization, but it is a part of the visualization system, performing tasks like volume loading and setting system parameters. The interaction method should be consistent with that of the virtual object manipulation so that once the program is started the same interface can be used to perform all tasks, instead of having to use mouse and keyboard for system control. Section 2.5 discusses how to map a $2 \mathrm{D}$ interface to the $3 \mathrm{D}$ environment. Using the method described, most system control can be performed in VEs.

\subsection{Volume Object Interaction}

In volume visualization, there is only one focused virtual object - the volume object and several other less focused virtual objects such as tools and setting widgets. Therefore, the use of more resources to manipulate the volume object is affordable. 
Besides the standard transformations discussed above, the volume object needs to be scaled universally or along individual axes. To study the volume, frequent rotation of the volume is required, so comfortable control is needed. Redundant control is also an advantage, so that a user can manipulate the volume object in the most convenient way. This section discusses methods to address the above problems.

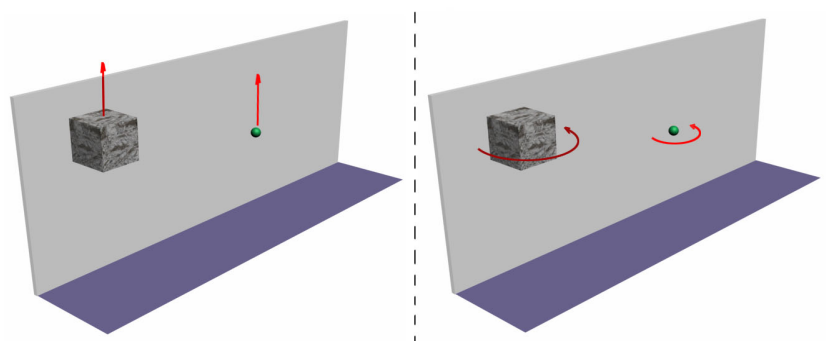

Fig. 2. The concept of the agent object

An agent object is proposed to manipulate the volume object. Besides directly manipulating the volume object, its translation and rotation can be controlled via the agent object. The agent object floats in a fixed position in space where it may be reached easily and manipulated by the user in a normal operating position. Fig. 2 illustrates this concept. A cuboid representing the volume object is shown in the projection screen above the floor, and the small sphere to the right is the agent object. When the user grabs the agent object and moves it, the volume object will move accordingly with the same direction and displacement. When the user releases the agent object, it will return to its default position, but the volume object will remain in its new position. This mechanism can be used to achieve cumulative translation (by ratchet translation). In the same manner, when the user rotates the agent object, the volume object will rotate accordingly around its own center; ratchet rotation can be achieved in the same way. Using the agent object, the user can always manipulate the volume object in a comfortable position, and is able to translate the volume object to a position not directly reachable (e.g. far behind the screen, or high above the head) if needed.

When visualizing volume data, users usually need to change the displayed size of the volume, for example shrinking the volume to have an overall view and enlarging the volume to observe local detail. In virtual environments, users have the option to do this by walking closer to enlarge the volume. (To be precise, the size of the volume object isn't changed, but the visual angle occupied by the volume is increased.) Similarly, walking further away shrinks the apparent volume (the visual angle is decreased). This is a very natural way to explore the volume object, just as in the real world. VRVolVis supports another way of changing the size of the volume object changing its actual size, either universally or along one axis. Using two virtual hands to grasp one of the cube edges bounding the volume object, the user can lengthen or shorten the cube along that axis by increasing or decreasing the inter-hand distance. If two adjacent edges are held, increasing or decreasing the inter-hands distance will scale the cube proportionally on the plane in which the two edges lie. If two non-adjacent edges are held, increasing or decreasing the inter-hand distance will 
scale the cube universally along all three axes. Fig. 3 illustrates the concept of two-handed scaling. The crosses represent the grabbed points on the edges of the volume object; the arrows show the directions of hand movements.

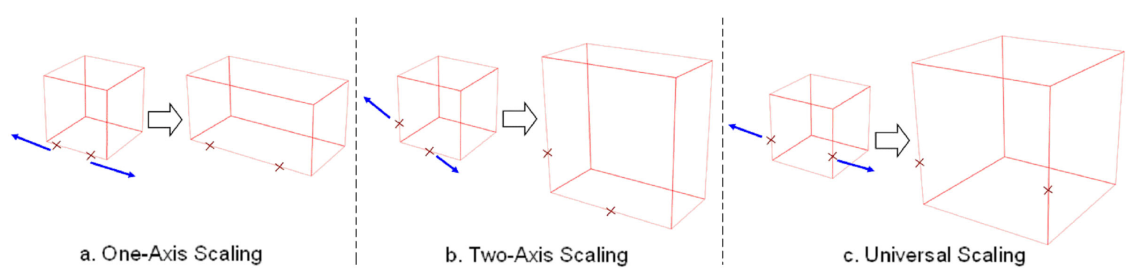

Fig. 3. The concept of two-handed scaling

\subsection{Transforming a 2D Interface from a 2D to 3D Environment}

For daily computer use, the WIMP interface is currently irreplaceable. Mouse and keyboard skills, for most people, are over-learned skills and most are quite adept at them. Furthermore, many concepts are developed and presented in 2D form - for example, the transfer function settings. For these reasons, the 2D interface can't be abandoned (at least immediately), so research on how to transform the 2D interface to the $3 \mathrm{D}$ environment is important.

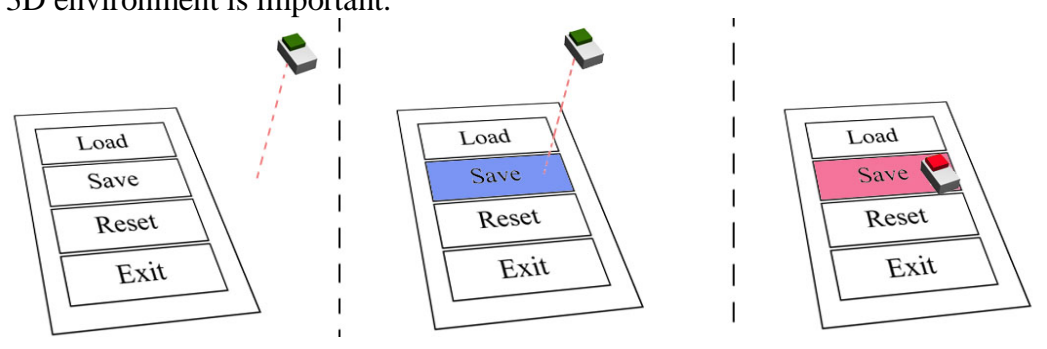

Fig. 4. The concept of transforming a $2 \mathrm{D}$ interface to a $3 \mathrm{D}$ environment

Schulze [22] suggested a method of transforming a 2D interface into 3D. The 2D interface elements, such as the menus and selectable items, are drawn on a 2D plane in 3D space; the user employs a handheld virtual laser pointer to operate the interface. However, the instability of the tracking system and human hand will be exaggerated using this method, and make accurate item selection very difficult. For VRVolVis, where the virtual scene is within reach, a ray-casting method is not necessary. To maintain consistency with other interaction paradigms, a design somewhat different to Schulze's is proposed, particularly in relation to item selection and operation methods. The menu can be handheld or float in space. When a virtual hand is close to the menu a light dotted line is shown, connecting the virtual index finger to the menu plane; the line is perpendicular to the plane. When the line intersects with a selectable item, the item will be highlighted. To select the item, the virtual hand is used to "press" the item (either by moving the virtual index finger to intersect the item or by pressing the 
switch attached to the thumb. Fig. 4 illustrates the concept.

For object moving tasks in a WIMP interface, such as moving a slider or moving an icon on a plane, usually a drag and drop metaphor is used. A basic object manipulation paradigm in VRVolVis is the grab and release metaphor, which can be seen as a $3 \mathrm{D}$ extension of drag and drop.

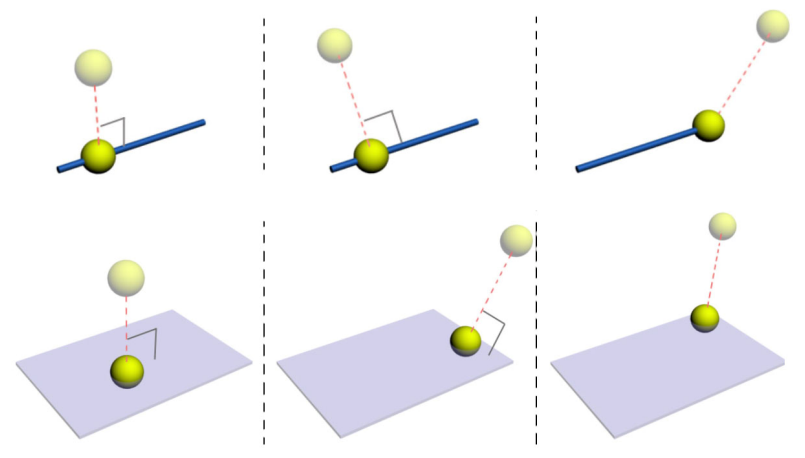

Fig. 5. The Ghost Object Paradigm for axial and 2D constraint

In VRVolVis, any movable item on a 2D widget (such as a menu) is represented by a 3D geometric object. To move it, the user can just grab it and move it. However, a motion constraint problem occurs in this situation. There is no physical constraint to limit hand movement in space, but there are many motion constraints in the VEs. For example, movable objects on a 2D widget should be constrained on the menu plane; sub-objects on a virtual tool should be constrained on the appropriate axis. Without proper visual feedback, the user may become confused about the interaction status. To solve this problem, a ghost object paradigm is proposed. When the object is within allowed range, the ghost object will not appear; when the user tries to move the object outside the allowed range, a ghost object (the same size as the controlled object, but shown with lower opacity) will follow the hand, providing visual feedback. The movable object's position is based on the projection of the ghost object position onto the menu plane. A dotted line connects the ghost object to the movable object. This line is normally perpendicular to the menu plane, but one end is clamped to stay within the menu plane so that the line stops being perpendicular if the hand is moved to a distant position. Once the user opens the fingers, the ghost object disappears. Fig. 5 illustrates the concept of the ghost object. The solid sphere represents the movable object; the axis and the plane represent the motion constraints; the faint sphere is the ghost object; and the rectangular marks denote perpendicular status.

\subsection{Some Interaction Conventions}

A good interface should inform users what functions of the system are available, instead of requiring them to remember all the commands. However, adding a few interaction conventions can improve the efficiency of the interface. A good example in a WIMP interface is the "right click" convention, which will bring forward a 
context-sensitive list related to the item that was clicked. Mine [11] proposed a few interaction conventions in VR, including the use of pull-down menus and over-the-shoulder deletion. He stated that three invisible menus can be placed over the user's head (left-top, top and right-top), and when a menu is needed, the user can just pull it down. The core of this idea is that the menus move with the user, having a fixed position relative to the user. Thus, the physical mnemonics of the user can be exploited. Over-the-shoulder deletion is used when a virtual object is no longer needed: the user can make it invisible using a motion like throwing it away over the shoulder. For VRVolVis, related but modified interaction conventions are proposed. An item-related menu is located in the right-behind position. A system menu is located in the left-behind position. We put the menu positions behind the user because this can further clarify the intention of grabbing a menu instead of, perhaps, a nearby virtual object. Over-the-shoulder deletion is used in VRVolVis also. This action will make a virtual object (a tool or a widget) invisible or put it back in its default position.

\subsection{Integration of the Techniques}

When the system starts, the volume object, agent object, and some virtual tools are floating in their default 3D positions. The user can then manipulate the volume object directly or via the agent object. To scale the volume object, the user uses the virtual hands to grasp two points on its edges to stretch or shrink it (as describe in Section 2.4). Fine control mode can be toggle on or off by touching a virtual box located in the lower right side of the VE space. Ghost object will appear whenever the user tries to move an object outside its allowed range. To use the exploration tools, the user can just take a tool and apply it to the volume object directly. System or object related menu can be brought out anytime by grabbing the left-behind or right-behind position, and the changes will be applied to the system immediately after an item is pressed. The proposed interaction techniques are thus integrated into one system.

\section{Evaluations}

Formal user studies will be performed to evaluate this interaction paradigm in our future research, but a pilot user study has already shown very positive results.

Eight subjects, all students or staff from Griffith University, were invited individually to the lab to use the prototype VRVolVis system to visualize some volumetric data sets. They all experienced 3 conditions: 1) Fixed-offset enabled; 2) Fixed- offset disabled; and 3) Agent object disabled.

After using the system, they were encouraged to talk about their experience and reactions. The following are some observations and subjective evaluations by these users. All considered the interaction paradigm very intuitive to learn and use. They all preferred the fixed-offset mode to the non-offset mode. Most, in fact, refused to use the non-offset mode after a few trials, stating they felt uncomfortable about the confused depth cues. When the agent object was enabled, most subjects preferred to use it rather than directly manipulating the volume object. All subjects were able to 
use the 2D menu in the VE to select desired items. Fig. 6 shows some of the many interaction tasks that the subjects were required to perform.

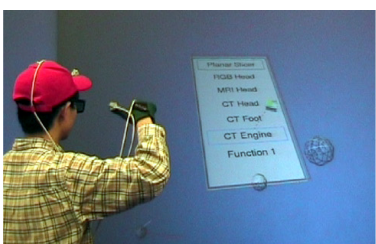

a)

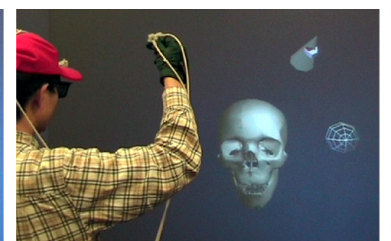

b)

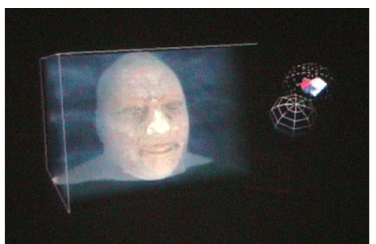

c)

Fig. 6. Some of the interaction tasks for the subjects: a) use the 3D menu to load different volume data sets; b) manipulate virtual objects using fixed-offset or non-offset direct manipulation; c) manipulate the volume object using the agent object.

\section{Conclusions}

A Human Computer Interaction paradigm for volume visualization in projection-based immersive virtual environments has been proposed. The paradigm included a number of novel interaction methodologies. A 3-state tracked glove proved to be a simple but powerful input device for VEs. A fixed-offset, direct manipulation method provided an effective method to overcome the problem of real hands occluding the closer-to-eye virtual objects in projection-based VEs. A fine control mode interaction method for direct manipulation was proposed to address the low accuracy problem of virtual object manipulation in VEs. An agent object interaction method was shown to be readily adopted by users, providing more flexibility and comfort in manipulating volume objects. A two-handed scaling method was proposed to conveniently scale volume objects along one, two, or three axes. A ghost object paradigm was proposed to address the motion constraint problem for the virtual objects. The interaction paradigm featured intuitiveness, high efficiency and accurate control for volume visualization in projection-based VEs, as demonstrated in early usability trials.

Acknowledgments. Many thanks to Dr. Mike Jones for his guidance, sharing of ideas and helping with development of the proposed HCI paradigm while he was a member of academic staff at Griffith University.

\section{References}

1. Lorensen, W.E. and H.E. Cline. Marching cubes: a high resolution 3D surface construction algorithm. in International Conference on Computer Graphics and Interactive Techniques. 1987: ACM Press.

2. Meissner, M., et al. A Practical Evaluation of Popular Volume Rendering Algorithms. in Volume Visualization Symposium. 2000. Salt Lake City.

3. Pfister, H., et al., The Transfer Function Bake-Off. Computer Graphics and Applications, IEEE, 2001. 21(3): p. 16-22. 
4. Zhang, S., et al. An Immersive Virtual Environment for DT-MRI Volume Visualization Applications: a Case Study. in IEEE Visualization. 2001. San Diego.

5. Souza, I., et al. Direct Volume Rendering of the Visible Human Dataset on a Distributed Multiprojection Immersive Environment. in Symposium on Virtual Reality. 2004. São Paulo.

6. Ware, C. and G. Franck, Evaluating stereo and motion cues for visualizing information nets in three dimensions. ACM Transaction on Graphics, 1996. 15(2): p. 121-140.

7. Ware, C. and S. Osborne, Exploration and virtual camera control in virtual three dimensional environments. ACM SIGGRAPH Computer Graphics, 1990. 24(2): p. 175-183.

8. Stoakley, R., M.J. Conway, and R. Pausch. Virtual reality on a WIM: interactive worlds in miniature. in the SIGCHI conference on Human factors in computing systems. 1995. Denver, Colorado, United States: ACM Press/Addison-Wesley Publishing Co.

9. Poupyrev, I., et al. The go-go interaction technique: non-linear mapping for direct manipulation in VR. in Symposium on User Interface Software and Technology. 1996. Seattle, Washington, United States: ACM Press.

10. Pierce, J.S. and R. Pausch. Comparing voodoo dolls and HOMER: exploring the importance of feedback in virtual environments. in the SIGCHI Conference on Human Factors in Computing Systems. 2002. Minneapolis, Minnesota, USA: ACM Press.

11. Mine, M.R., J. Frederick P. Brooks, and C.H. Sequin. Moving objects in space: exploiting proprioception in virtual-environment interaction. in International Conference on Computer Graphics and Interactive Techniques. 1997: ACM Press/Addison-Wesley Publishing Co.

12. Koutek, M., Scientific Visualization in Virtual Reality: Interaction Techniques and Application Development, in Faculty of Electrical Engineering, Mathematics and Computer Science. 2003, Delft University of Technology: Delft, the Netherlands. p. 251.

13. Schulze, J.P., et al. Volume Rendering in a Virtual Environment. in the 5th IPTW and Eurographics Virtual Environments. 2001: Springer Verlag.

14. Kitware Volview 2.0, http://www.kitware.com/products/volview.html.

15. Kniss, J., et al., Interactive texture-based volume rendering for large data sets. IEEE Computer Graphics and Applications, 2001. 21(4): p. 52-61.

16. Guiard, Y., Asymmetric Division of Labor in Human Skilled Bimanual Action: The Kinematic Chain as a Model. The Journal of Motor Behaviors, 1987. 19(4): p. 486-517.

17. Haan, G.d., M. Koutek, and F.H. Post. Towards intuitive exploration tools for data visualization in VR. in the ACM symposium on Virtual reality software and technology. 2002. Hong Kong, China: ACM Press.

18. Stephenson, P., et al. Studydesk: Semi-Immersive Volumetric Data Analysis. in the 1st international conference on Computer graphics and interactive techniques in Australasia and South East Asia. 2003. Melbourne, Australia: ACM Press.

19. Bowman, D.A. and L.F. Hodges, Formalizing the Design, Evaluation, and Application of Interaction Techniques for Immersive Virtual Environments. Journal of Visual Languages and Computing, 1999. 10(1): p. 37-53.

20. Bowman, D.A., Interaction Techniques for Common Tasks in Immersive Virtual Environments, in Department of Computer Science. 1999, Georgia Institute of Technology: Atlanta, Georgia. p. 132.

21. Bowman, D.A. and L.F. Hodges. An evaluation of techniques for grabbing and manipulating remote objects in immersive virtual environments. in Symposium on Interactive 3D Graphics. 1997. Providence, Rhode Island, United States: ACM Press.

22. Schulze-Döbold, J., Interactive Volume Rendering in Virtual Environments, in Institute for Visualization and Interactive System. 2003, University of Stuttgart: Stuttgart, Germany. p. 157. 